\title{
Characterization of Doppler Effects in the Context of Over-the-Horizon Radar
}

\author{
Cornel Ioana \\ Grenoble Institute of Technology \\ GIPSA-lab, Signal-Image Department \\ Saint Martin d'Hères, France \\ cornel.ioana@gipsa-lab.grenoble-inp.fr
}

\author{
Moeness G. Amin, Yimin D. Zhang, Fauzia Ahmad \\ Villanova University \\ Center for Advanced Communications \\ Villanova, PA 19085, USA \\ [moeness.amin, yimin.zhang, fauzia.ahmad]@villanova.edu
}

\begin{abstract}
This paper addresses the problem of the characterization of Doppler effect of maneuvering targets in the context of over-the-horizon radar. The received signal has a complex Doppler structure which is composed of several arrivals, each corresponding to a particular path. In essence, it consists of several close time-frequency components with non-linear signatures in the time-frequency domain. The nonlinearities are the projections of the target's motion vectors on the propagation paths. Estimating the time-frequency contents of all paths reveals the Doppler effects characterizing the target's trajectory. Analysis of such signals in the presence of strong clutter requires effective non-stationary signal processing techniques. In this paper, we propose a new technique based on local analysis of the phase information using warped high-order ambiguity function. The results depict resolvable multipath estimates which are very close to the ground truth.
\end{abstract}

\section{INTRODUCTION}

Radar signal returns from maneuvering targets are typically examined in the frequency domain using Doppler filters and moving target indication techniques [1]. This is achieved by applying Fourier analysis to the received data for each range gate and forming a detector incorporating the output of each filter bank. However, rapidly moving targets within the same range gate causes time-varying Doppler signatures. This gives rise to non-stationary signal returns whose power is distributed in both time and frequency. As such, application of conventional frequency decomposition techniques leads to loss of local behavior and temporal frequency information. Therefore, if the aim is to characterize target instantaneous velocity as well as spatial position and direction of motion, joint time-frequency signal representation and processing should be used in lieu of the traditional Fourier transform [2].

In over-the-horizon radar (OTHR) applications, the waveform is narrowband and emitted from an antenna array with phase coherence so as to point the energy in various directions. The returned signal from a moving target has time-varying frequency contents [3]. One of the challenges is to detect the target's presence and determine its spatial position over time. This is achieved based on the target's time-dependent Doppler information which presents itself in a localized signature in the time-frequency domain. Unknown target motion profiles, including acceleration and complex maneuverability, in the presence of multipaths, limit the use of physical models and call for the application of nonparametric estimation methods for multi-component signals.

In this paper, we consider estimation of moving target parameters in OTHR by utilizing the time-frequency-phase continuity of each Doppler component. That is, despite its arbitrary non-linearity due to the unknown target motion, a Doppler modulation corresponding to one path has a continuous phase variation that could be exploited for accurate tracking. To do so, the local phase is analyzed using the warped third-order ambiguity function [4]. Furthermore, local third-order polynomial phase components are merged using local correlation maximization. The tracking Doppler component is then extracted using a time-frequency filter. The process is iterated until all Doppler components are estimated.

The paper is structured as follows. Section II briefly recalls the model of the Doppler effect in the OTHR context. The proposed estimation method is described in Section III. Results based on simulated data, illustrated in Section IV, highlight and support the potential of the proposed method.

\section{MODEling THE DOPPlER EFFECT IN OTHR CONTEXT}

Let us assume, as proposed in [5], that the propagation paths of the emitted/received signals are defined as shown in Fig. 1, where the received signal consists of three components. The first and second components correspond to both the emitted and received signals propagating along path I and path II, respectively. The third component is a result of the emitted signal traveling along path I and the received signal propagating along path II or vice versa. In Fig. $1, H$ is the height of the ionosphere layer, and $h$ is the height of the target.

Under this assumption, the received signal can be expressed as follows:

$$
x(t)=A_{1} e^{j \varphi_{1}(t)}+A_{2} e^{j \varphi_{2}(t)}+A_{3} e^{j \varphi_{3}(t)},
$$

where $A_{1}, A_{2}$ and $A_{3}$ are the path losses, and $\left\{\varphi_{i}\right\}$ 's are the instantaneous phase laws, which can be expressed as [5] : 


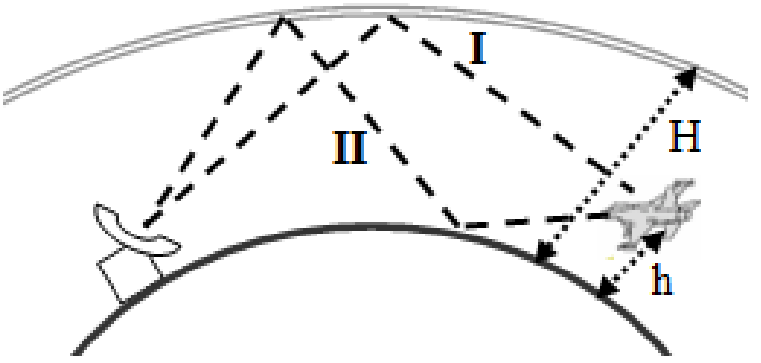

Figure 1. Definition of the propagation paths in the OTHR [5]

$$
\begin{aligned}
& \varphi_{1}(t)=-\frac{4 \pi f_{c}}{c} \int\left[K(t) v_{R}(t)-\frac{2 H}{R(t)} v_{c}(t)\right] d t \\
& \varphi_{2}(t)=-\frac{4 \pi f_{c}}{c} \int\left[K(t) v_{R}(t)+\frac{2 H}{R(t)} v_{c}(t)\right] d t \\
& \varphi_{3}(t)=-\frac{4 \pi f_{c}}{c} \int K(t) v_{R}(t) d t
\end{aligned}
$$

In eq. (2), $K(t)=\left(1-2 H^{2} / R^{2}(t)\right), v_{R}(t)=d R(t) / d t$ is the target velocity in the range direction, and $v_{c}(t)=d h(t) / d t$ is the velocity of the target along the vertical position.

Under these assumptions, the time-frequency content of the received signal is composed of three non-linear timefrequency signatures, very close to each other. For the scenarios proposed in [5], where a target makes a $180^{\circ}$ turn in approximately 31 seconds to change height and direction, the instantaneous frequency laws (IFLs) of the components in eq. (2) are illustrated in Fig. 2. Such signal will be used in the next section to define the time-frequency-phase methodology that will provide the IFL estimation of each path-dependent Doppler variation.

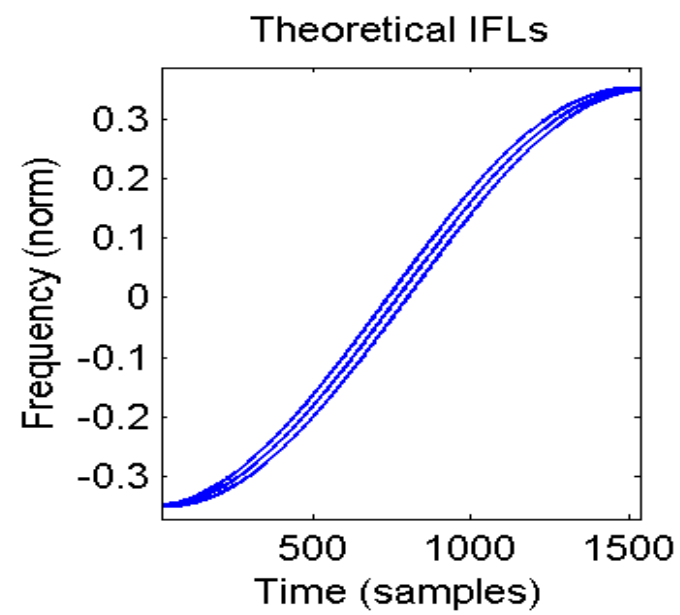

Figure 2. Time-varying Doppler signatures of a received baseband signal (initial range $=2000 \mathrm{~km}$, height of ionosphere layer $H=350 \mathrm{~km}$, initial target height $h(0)=10 \mathrm{~km}$, carrier frequency $f_{c}=20 \mathrm{MHz}, 256$ samples/block)

\section{TIME-FreQUenCy TRACKING USING LOCAL PhaSE MODELING}

Generally, radar signals propagated in a multipath environment and backscattered from moving targets are characterized by several time-frequency components having non-linear IFLs. Such a signal has been defined in the previous section. Fig. 3(a) shows the spectrogram's limits; when the time-frequency components are close, it is not possible to clearly visualize the three components. This is a consequence of the tradeoff between time and frequency resolutions and it could affect any time-frequency tracking method based on short-time Fourier transform.

An alternative to this type of methods is the local analysis of time, frequency, and phase coherence, and to use this information to merge local components in order to get the global time-frequency structures characterizing the signal. The first step of this methodology is the short-time polynomial phase modeling of order 3. As the signal is characterized by non-linear heterogeneous time-frequency components (see Fig. 2 for example), its short-time analysis is a natural way to represent efficiently the time-frequency content of the signal. Approximating the local time-frequency content by Gabor atoms or by linear chirps has been analyzed in previous works (see [6] for a synopsis). In our approach, we propose to use the cubic frequency modulations (FMs) in order to better approximate the non-linear parts of the signal's IFLs. In addition, in using cubic FM for short-time signal's phase modeling, the window size is less important than in the case of linear chirps.

In our context, the short-time polynomial phase modeling of order 3 is given by warped high-order ambiguity function (WHAF) [7]. This analysis is done in adjacent windows, half-overlapped, as illustrated in the Fig. 3(b).
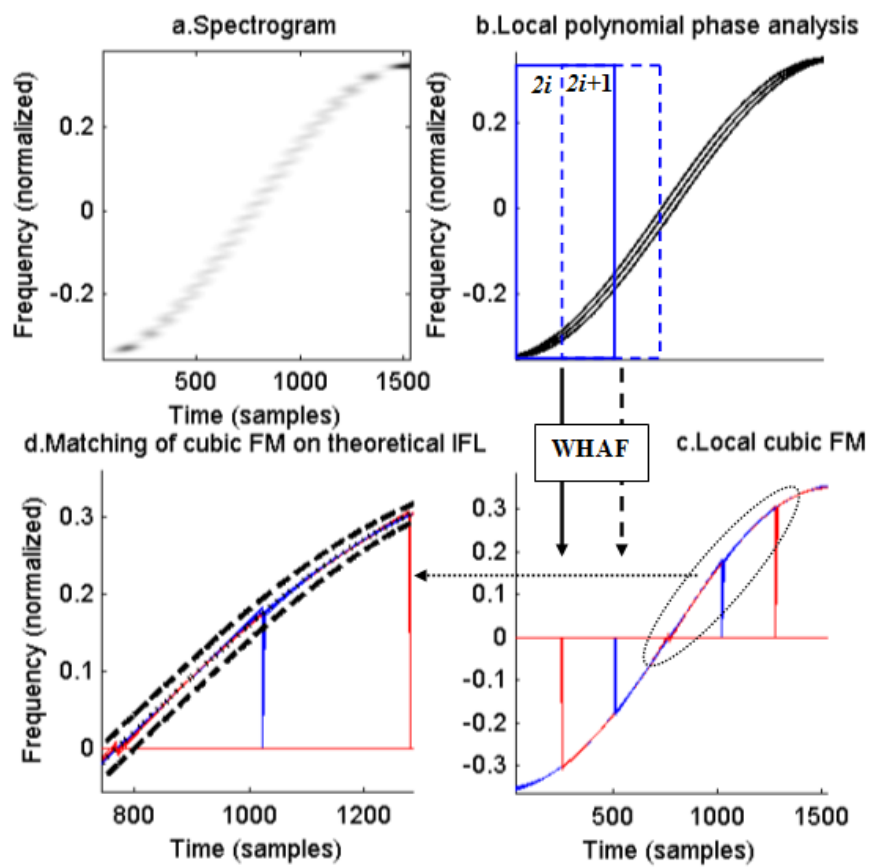

Figure 3. Short-time WHAF in half over-lapped windows 
For each window position, the WHAF of order 3 is applied to provide the third-order phase modeling of the components. The IFL estimates obtained for two (overlapped) neighboring windows, $2 i$ and $2 i+1$, are plotted in Fig. 3(d) (for two windows of 512 samples). We observe that the IFLs are well fitted to the modeled components. Indeed, in real situations, this modeling is affected by noise and/or by close components. For this reason, WHAF provides, for each window, several estimations of the same component. Consider $N_{c}$ estimates for each window. Let us denote the set of phase functions obtained from WHAF-based phase modeling applied in the $i$ th window as :

$$
D^{(i)}=\left\{\psi_{k}^{(i)}\right\}_{k=1, \ldots, N c}
$$

where $\psi_{k}^{(i)}$ is the $k$ th phase function of order 3 defined as:

$$
\psi_{k l}^{(i)}(t)=\sum_{l=1}^{3} a_{k l} t^{l} ; t \in[i T ;(i+3 / 2) T]
$$

and $T$ is the window size.

As indicated in Fig. 3, the phase functions are just an approximation of the real time-frequency content of the signal. For this reason, they are used for regrouping procedure provided by the second step of the methodology fusion of local time-frequency-phase information. The phase functions (4) are used to build local filter functions which extract the signal's samples corresponding to the timefrequency regions defined in the neighborhood of the local functions [8]. The design of three such filters is illustrated in Fig. 4 for the region corresponding to the interval from sample number 700 to 820 .
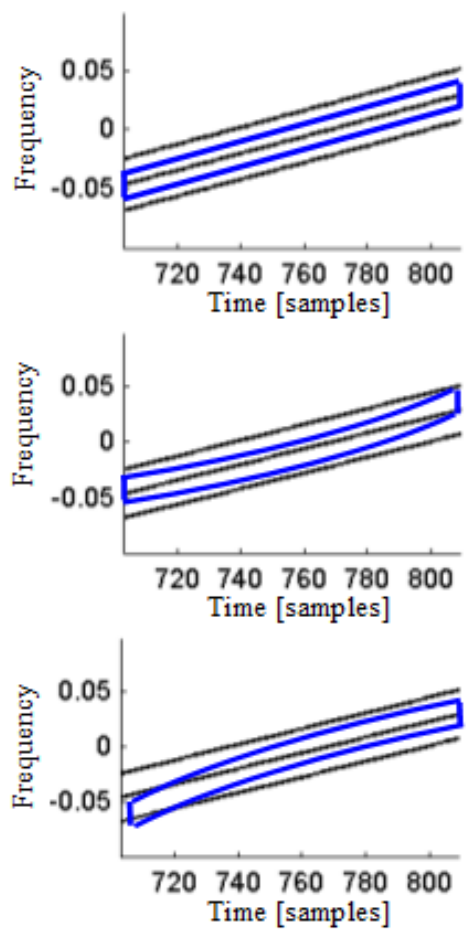

Figure 4. Local time-frequency filters defined from $\psi_{k}^{(i)}$ function (only three functions are illustrated)
Let us consider signal $x$ at two analyzing windows $2 i$ and $2 i+1$ (as illustrated in Fig. 3). Consider $D^{(2 i)}=\left\{\psi_{k}^{(2 i)}\right\}_{k=1, \ldots, N c}$ and $D^{(2 i+1)}=\left\{\psi_{k}^{(2 i+1)}\right\}_{k=1, \ldots, N c}$, the sets of phase functions provided by the WHAF for the $2 i$ th and the $(2 i+1)$ th windows, respectively. Using these phase functions, we build, as indicated in Fig. 4, the sets of time-frequency filters $\left\{W_{k}^{(2 i)}\right\}_{k=1, . ., N c}$ and $\left\{W_{k}^{(2 i+1)}\right\}_{k=1, . ., N c}$. With the help of these filters, we extract, from the analyzed signal $x$, the corresponding signal's samples denoted by:

$$
\begin{aligned}
& \left\{s_{k}^{(2 i)}\right\}=\left\{\left(W_{k}^{(2 i)} x\right)(t)\right\}_{k=1, . ., \text { Ncomp }} \\
& \left\{s_{k}^{(2 i+1)}\right\}=\left\{\left(W_{k}^{(2 i+1)} x\right)(t)\right\}_{k=1, . ., \text { Ncomp }}
\end{aligned}
$$

Using these samples, we decide that two phase functions, $\psi_{m}^{(2 i)}$ and $\psi_{n}^{(2 i+1)}$, are regrouped (i.e., they belong to the same T-F component) if the correlation of corresponding samples, $\left\{s_{m}^{(2 i)}\right\}$ and $\left\{s_{n}^{(2 i+1)}\right\} ; m, n=1, \ldots, N c$, is maximal for all pairs $(m, n)$ :

$$
\begin{aligned}
& (\hat{m}, \hat{n})=\max _{\substack{m \in[1, N c] \\
n \in[1, N c]}}\left|\left\langle s_{m}^{(i)}(t), s_{n}^{(i+1)}(t)\right\rangle\right| \Rightarrow \\
& \Rightarrow \psi_{\hat{m}}^{(i)} \circ \psi_{\hat{n}}^{(i+1)} \subset \text { Trajectory } \\
& \text { TF } \phi_{j}[(i-1) T:(i+1) T] \text { of signal } x
\end{aligned}
$$

where "o" symbolizes the fusion of two phase functions belonging to the same trajectory defined in the time interval $[(i-1) T,(i+1) T]$ of the $j$ th component of the signal. After studying all segments of the signal, we define the timefrequency trajectory corresponding to the $j$ th component of the signal as the fusion of phase functions following (6):

$$
\phi_{j}(t)=\psi_{k_{1}}^{(1)} \circ \psi_{k_{2}}^{(2)} \circ \ldots \circ \psi_{k_{i}}^{(i)} \circ \ldots \circ \psi_{k_{N}}^{(N)}
$$

where $N$ is the number of analyzing windows and $k_{i}$ is the index of the phase function obtained from the $i$ th window.

The time-frequency trajectory $\phi_{j}(t)$ is used to design the time-frequency filter that will extract the $j$ th component of the signal. This constitutes the third step of the methodology global time-frequency filtering. For the signal shown in Fig. 2 , the first estimated trajectory corresponds to the middle component. Around this trajectory, we build the timefrequency filter [8] that will extract the samples of the corresponding component. We remark that the residual signal contains the remaining two components in (2), proving the accuracy of the filtering procedure (Fig. 5(a)). At the next iteration, the residual signal (Fig. 5(b)) is processed in the same way until all trajectories are estimated (Fig. 5(c)).

Consequently, the iterative application of the three steps of the time-frequency-phase methodology allows extracting individual components of the analyzed signal. 


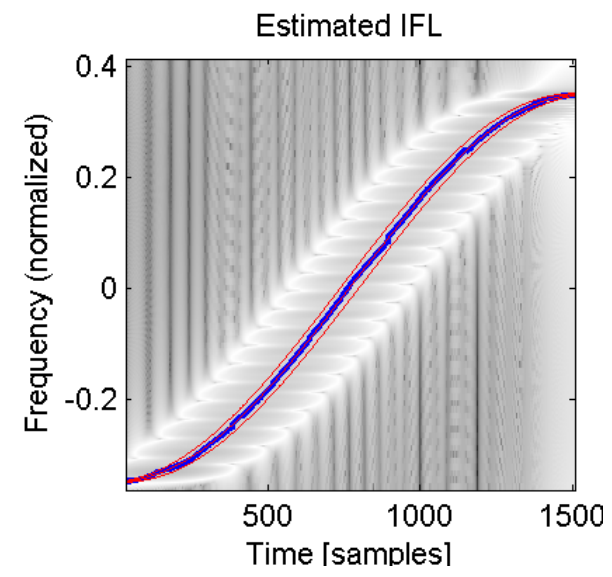

a. The tracking of the IFL of the third component in (2)

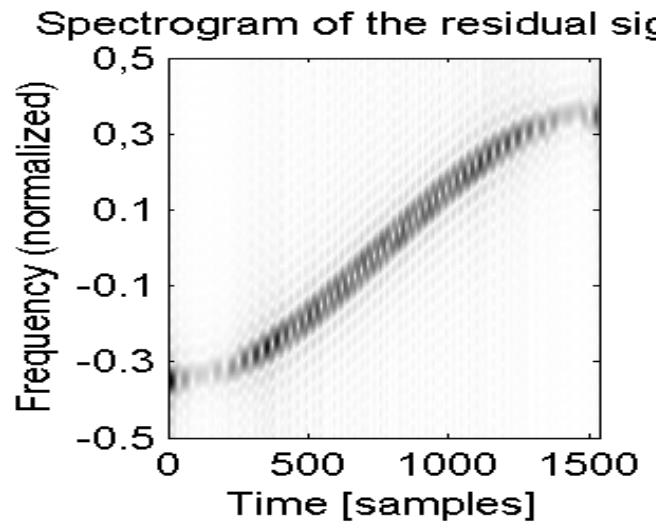

b. Remaining components after the first filtering

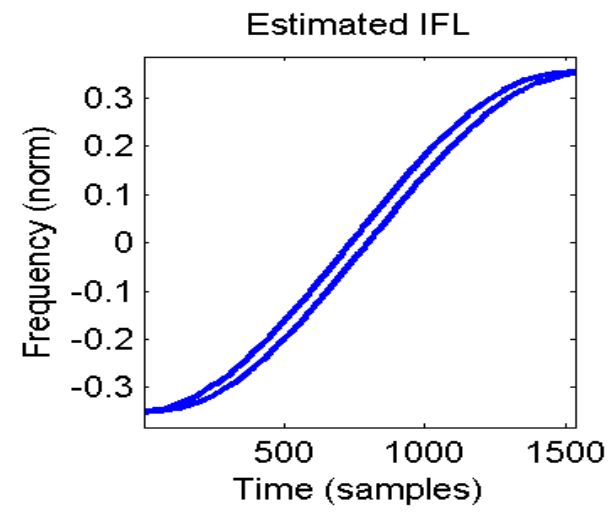

c. The tracking of the IFL of the first and second component in (2)

Figure 5. Global time-frequency filtering and estimation of the remaining components

\section{RESULTS}

The accuracy of the extracted method is provided, for the scenario described in Fig. 1, in Fig. 6 where we plot the errors between theoretical and estimated IFLs. We can observe that the estimation errors are very small, except at the borders of the windows. But even in these regions the errors are acceptable which can be of great help for further estimation of the target's trajectory.

In the next example we consider the tracking of IFLs in the presence of real clutter. The configuration is the same as the one defined in Fig. 2. The signal-to-clutter ratio (SCR), after prewhitening processing, is $-11.2 \mathrm{~dB}$. The spectrogram of the received signal is plotted in Fig. 7. We remark that the clutter, concentrated at low frequencies, has higher energy than the signal.
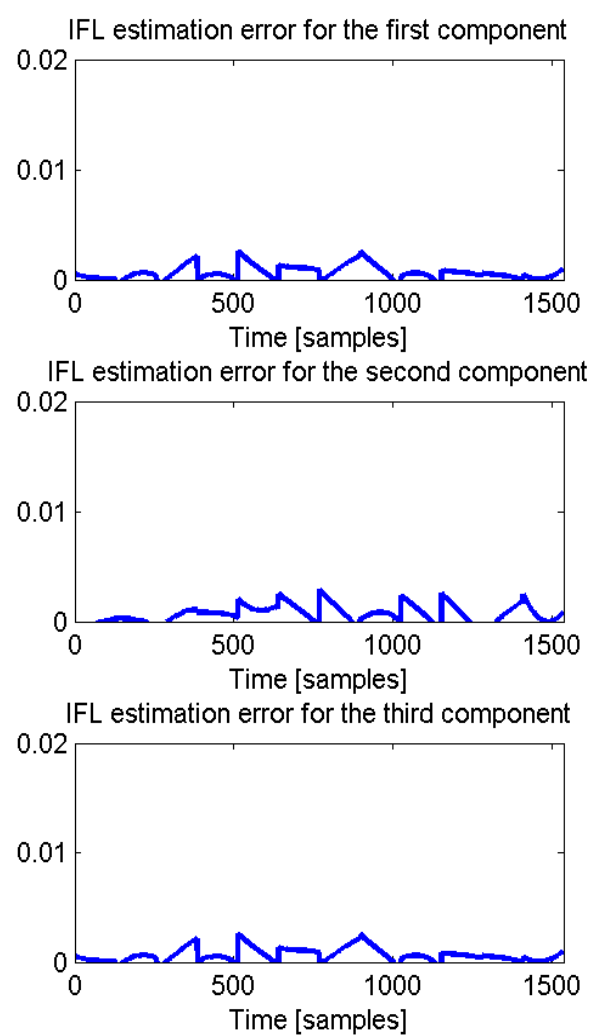

Figure 6. IFL estimation accuracy

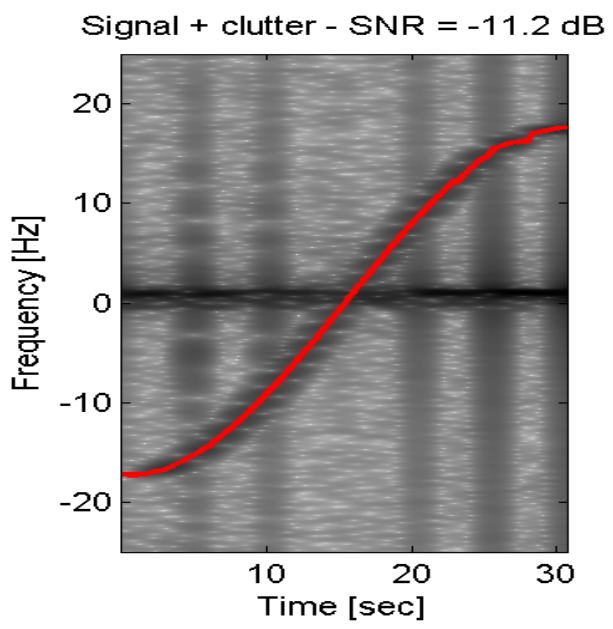

Figure 7. Spectrogram of the noised signal and the estimated IFL of the middle component 
For this example, the IFL of the third component in (2) is accurately tracked in spite of the high level of clutter. This interesting result is explained by the local analysis of the phase using the WHAF of order 3. More precisely, modeling the instantaneous phase by a cubic FM can produce a natural separation of the signal's components from the clutter as indicated in Fig. 8 in the context of the local phase modeling of a segment of 256 samples.

\section{a. WHAF of order 3 b. WHAF of order 2}
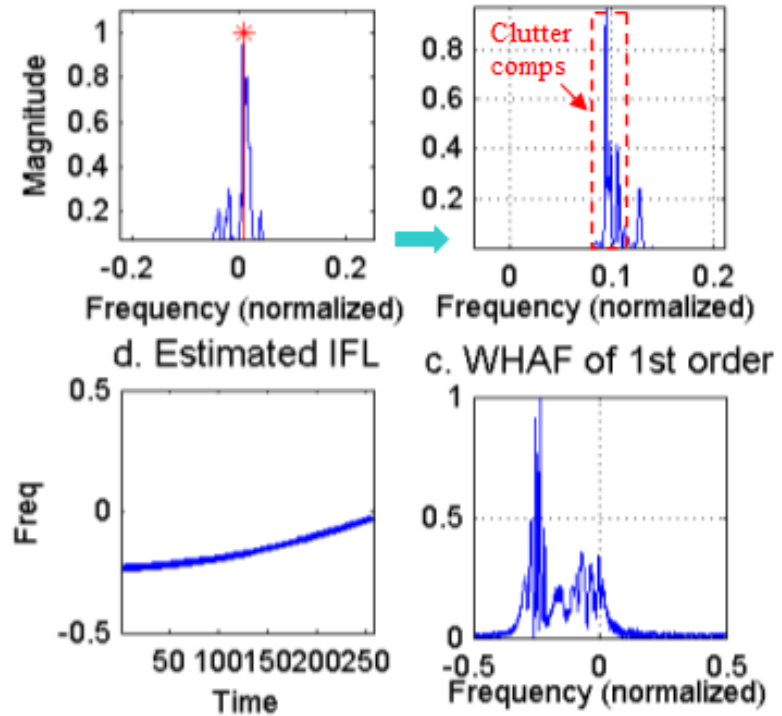

Figure 8. Separation of signal's component from clutter, using high-order ambiguity function

The WHAF based modeling starts with order 3, as indicated in Fig. 8(a). Once the third-order coefficient is estimated, $a_{3}$, its contribution is eliminated by multiplying the signal with the corresponding cubic FM $\exp \left(-j 2 \pi a_{3} t^{3}\right)$. After this multiplication, the WHAF of order 2 is applied (Fig. 8(b)). In the domain of the WHAF of order 2, the separation of signal's component from the clutter is possible. Actually, while the clutter is mainly concentrated around the origin (zero frequencies), the chirp rates necessary to model such components are small which is materialized by strong second-order polynomial coefficients (see the dashed rectangle in Fig. 8(b)). The coefficients are stronger because of the high clutter energy, but the fact that they are located around low frequencies allows us to separate them. Indeed, the second-order polynomial coefficients that will be taken into account in the local phase modeling are located out of the region defined around the origin. Furthermore, the estimation of the first-order coefficient (Fig. 8(c)) as well as the other polynomial coefficients (of order 2 and 3 ) will lead to an accurate estimation of the IFL, despite the noise.

This interesting property explains the good tracking performances in the presence of the clutter. The accurate estimation of the IFL is indicated in Fig. 7 and it could be further used to extract the corresponding signal's component and modeling the next two components. Proceeding in this way, we can obtain the Doppler component, thus enabling estimation of the target's trajectory.

\section{CONCLUDING REMARKS}

In this paper, we developed a new approach for resolving and tracking multi-component Doppler signals arising due to multipath propagation in the context of OTHR. The approach is based on time-frequency-phase coherence of the Doppler signal which is locally exploited by polynomial phase modeling of order 3 .

The results proved the efficiency of the proposed methodology. The simultaneous availability of the timefrequency and phase information (by local polynomial phase modeling of order 3) allows us to follow all time-Doppler frequency variations, in spite of closeness of the various components and presence of noise interferences. The timefrequency-phase information acts as a continuity criterion preserving the time-frequency structure of each component. After one component has been tracked, its extraction via a global time-frequency filtering is performed. In this way, the extraction is independent of the component's amplitude.

\section{ACKNOWLEDGMENT}

The work by C. Ioana was performed at Villanova University under ONR Global VSP, grant no. N62909-09-1-4082.

\section{REFERENCES}

[1] M. I. Skolnik (ed.), Radar Handbook, 2nd edition, New York, NY: McGraw-Hill, 1990.

[2] V. Chen and H. Ling, Time-Frequency Transforms for Radar Imaging and Signal Analysis, Boston, MA: Artech House, 2002.

[3] A. A. Kolosov, Over-the-Horizon Radar, Boston, MA: Artech House, 1987.

[4] C. Ioana and A. Quinquis, "Time-frequency analysis using warped-based high-order phase modeling", EURASIP Journal of Applied Signal Processing, vol. 2005, no. 17, pp. 2856-2873, September 2005.

[5] Y. Zhang, M. G. Amin, and G. J. Frazer, "High-resolution time-frequency distributions for manoeuvring target detection in over-the-horizon radars", IEE Proc.-Radar Sonar Navig., vol. 150, no. 4, pp. 299-304, August 2003.

[6] A. Papandreou-Suppappola (ed.), Applications in TimeFrequency Signal Processing, Boca Raton, FL: CRC Press, 2003.

[7] C. Ioana and A. Quinquis, "Time-frequency analysis using warped-based high-order phase modeling," EURASIP Journal of Applied Signal Processing, vol. 2005, no. 17, pp. 2856-2873, September 2005

[8] A. Jarrot, C. Ioana, and A. Quinquis, "Toward the use of the time-warping principle with discrete-time sequences," Journal of Computers, vol. 2, no. 6, pp. 4955, August 2007. 